\title{
Examination versus subjective nasal obstruction in the evaluation of the nasal septal deviation*
}

\author{
Murat Salihoglu', Engin Cekin', Aytug Altundag², Enver Cesmeci \\ ' Otolaryngology Department of GATA Haydarpasa Training Hospital, Istanbul, Turkey \\ ${ }^{2}$ Otolaryngology Department of Istanbul Surgery Hospital, Istanbul, Turkey
}

Rhinology 52: 122-126, 2014

DOl:10.4193/Rhino13.057

*Received for publication:

May 6, 2013

Accepted: November 24, 2013

\begin{abstract}
Background: Nasal septal deviation (NSD) is a frequent complaint among patients in an otolaryngology clinic. The prevalence of NSD varies in different populations and NSD classification schemes are complex.

Methods: We aimed to determine the prevalence of NSD in a populationof almost ten thousand Turkish young males using a new NSD classification method that we developed. We compared the results with patients' complaints. All patients underwent two nasal examinations, which were performed using a nasal speculum with and without administration of vasoconstrictor agents. Inferior Concha Hypertrophy $(\mathrm{ICH})$ was evaluated in the first examination and NSDs were scored during the second examination. All findings were recorded according to our classification scheme. Severity of nasal obstruction was subjectively evaluated by using a visual analogue scale (VAS).
\end{abstract}

Results: We found a significant association between prevalence of NSD and nasal injuries in over $8 \%$ of the patients, but there was no association between the mode of delivery and prevalence of NSD. Surprisingly, about $30 \%$ of the participants with NSD had no complaint of nasal obstruction. Although most of the participants in the study had no severe nasal obstruction complaint, we found an association between NSD presence and patient's VAS.

Conclusion: NSD is very frequent in the Turkish population and most often related to trauma; however, its effect on patient quality of life varies.

Key words: nasal, septum, deviation, concha, hypertrophy

\section{Introduction}

Nasal septal deviation (NSD) and inferior concha hypertrophy (ICH) commonly cause nasal obstruction, which is a frequent complaint among patients in an otolaryngology clinic ${ }^{(1)}$. In the literature, the prevalence of NSD varies in different age groups (from newborn to adults) ranging from $0.93 \%$ to $76.2 \%(2,3)$. Prior NSD classification schemes may be complex and time-consuming especially for the purpose of prevalence studies. These classification methods also incompletely reflect the negative effects of NSD and other present nasal pathologies on respiration ${ }^{(1,3-5)}$. Classification systems that are simple and easy to apply are needed, especially in prevalence studies. In this study, we primarily aimed to determine the prevalence of NSD in a population of Turkish young males using a new NSD classification method that we developed and describe in this article. We scored the complaints of the patient and evaluate correlations between findings in the examination and complaints. We also evaluated the relation of NSD with trauma history and kind of labour.

\section{Materials and methods}

\section{Patients}

A total of 9,835 young males underwent otolaryngological 
examination as part of a legal requirement prior to participate in heavy physical activity. Patients with a history of nasal surgery, nasal cavity tumour, allergic rhinitis, nasal polyps and active respiratory infection were excluded. Patients were evaluated between January 2010 and June 2011. The Medical Ethics Committee of GATA Haydarpasa Training Hospital approved the study.

\section{Nasal examination}

Nasal examinations were performed by the two experienced otolaryngologists. Initial nasal examination was performed on each patient by using a nasal speculum without previous administration of vasoconstrictor agents and local anaesthetics. After that, $1 \mathrm{mg} / \mathrm{ml}$ xylometazoline hydroclorur spray (Otrivine ${ }^{\circledR}$ spray, Novartis, Luleburgaz, Turkey) was applied once topically to both nostrils and two minutes after administration of the nasal spray, the nasal examination was repeated. Inferior concha hypertrophy was evaluated and scored in the first examination but septal deviations were scored according to the findings on the second examination to see posterior deviations, which were not seen on the initial examination because of $\mathrm{ICH}$. Findings were recorded according to our classification procedure described in Figure 1. The designated score for turbinate hypertrophy was 1 if present, and absence of this finding was scored as 0 . The degree of NSD was scored based on the degree of nasal cavity regardless of its location (being posterior or anterior) in the nasal cavity. One point was given if NSD was covering one third of the nasal cavity, 2 points if it was covering two third, and 3 points if it was totally obstructing the whole nasal cavity. The total score was calculated by adding the scores of each nasal cavity. After these steps all the patients were informed about their attending status to the training. This way, we were sure that they knew about their status, the questionnaire or anything that would be done after this step, won't change their status. Then using the visual

Table 1. Questionnaire that we used for participants.

Please answer the following questions:

1) History of nasal injury

a) yes

b) No

2) Kind of labor

a) Caesarean section delivery

b) Spontaneous vaginal delivery

3) 0 indicates that the nose is completely open and 10 indicates that the nose is completely close. How well is your breathing? Please mark.

$0 \ldots \ldots 1 \ldots \ldots 2 \ldots \ldots 3 \ldots \ldots 4 \ldots \ldots 5 \ldots \ldots 6 \ldots . \ldots \ldots 8 \ldots \ldots 9 \ldots \ldots 10$ (VAS)

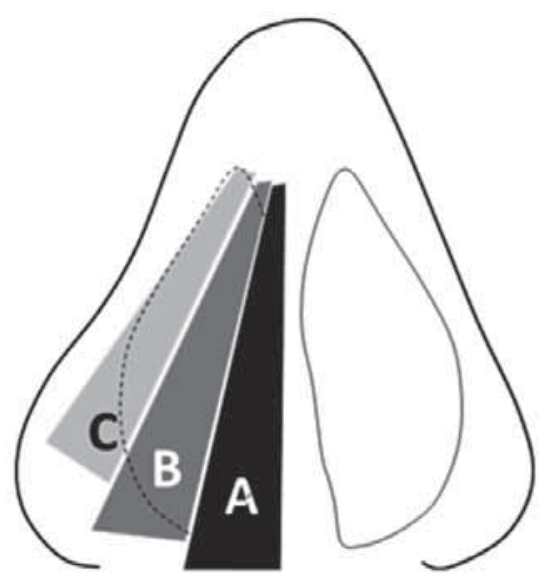

Figure 1. NSD can cause one-third obstruction of the nasal cavity (A), two-third obstruction of the nasal cavity (B) or complete obstruction of the nasal cavity (C) and it is scored 1,2 or 3, subsequently. ICH is evaluated and marked as present or absent.

analog scale (VAS), patients were asked to score their degree of nasal obstruction from 0 (no obstruction) to 10 (total obstruction). Patients were also asked to fill a questionnaire about method of delivery (vaginal versus caesarean section) and presence of nasal injury (Table 1).

\section{Statistical analysis}

For data input, we used MS Excel 2010 (Microsoft Corp.). We also used IBM SPSS statistics Version 20.0.0 (Chicago, IL, USA) for statistical analysis. Descriptive statistical methods for univariate data analysis were used.

\section{Results}

A total of 9,835 young males between the ages of 20 and 29 years (mean age $21.71 \pm 2.23$ ) were included in the study. NSD was identified in 4,580 (46.56\%) and ICH was identified in 895 (9.10\%) of them. There was unilateral NSD in 2,746 patients (59.96\% of all septal deviations) and bilateral NSD in 1834 (40.04\% of all septal deviations). NSD was on the left side in $1,352(49.23 \%)$ and on the right side in 1,394 (50.77\%) in those patients with unilateral NSD. ICH was only on the left side in 385 (43.01\%) patients, on the right side in 283 (31.62\%) patients, and bilateral in 227 (25.37\%) of the patients with ICH. ICH was identified in 784 (17.11\%) of the patients who had NSD and 111 (2.11\%) of the patients who did not have NSD. As a result, we found that ICH was significantly higher in patients with NSD compared to patients without NSD $(p<0.001)$.

A total of 385 patients with NSD (8.40\%) and $36(0.78 \%)$ patients without NSD had a history of nasal trauma. It indicates a statistically significant correlation between the prevalence of NSD and nasal trauma $(p<0.001)$. A total of $4,182(91.31 \%)$ patients 


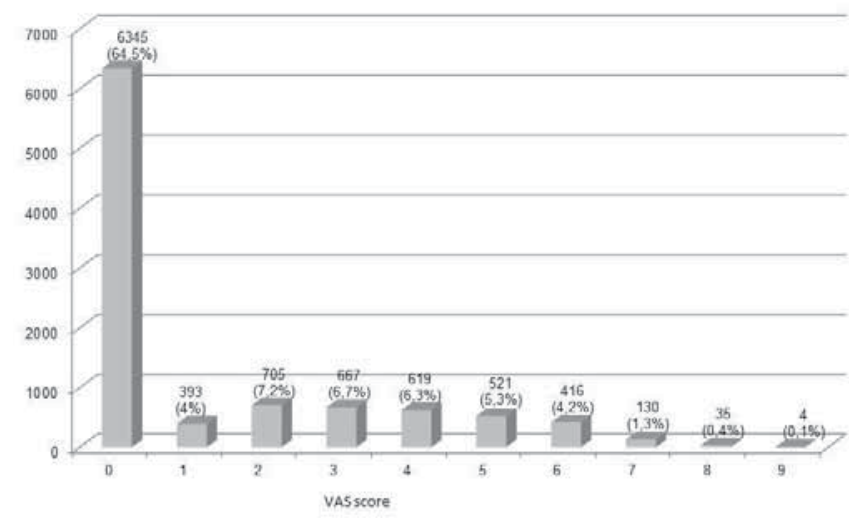

Figure 2. VAS values of the young male population.

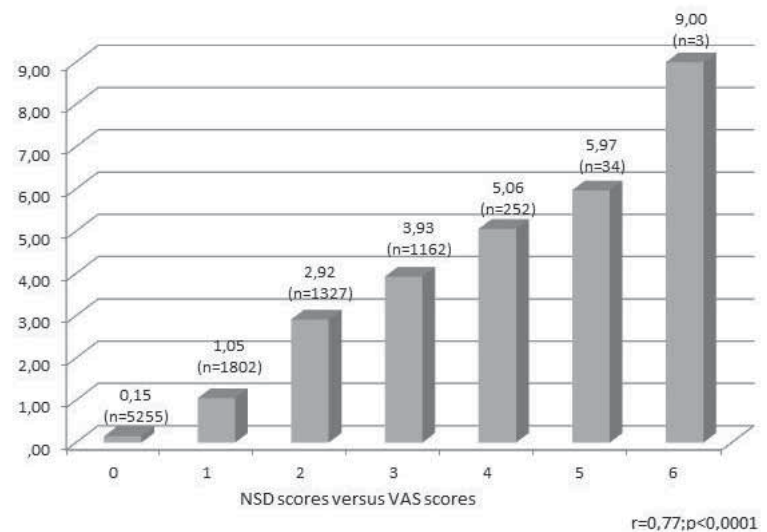

Figure 3. The distribution of NSD and VAS scores. NSD: Nasal Septum Deviation; VAS: Visual Analogue Scale.

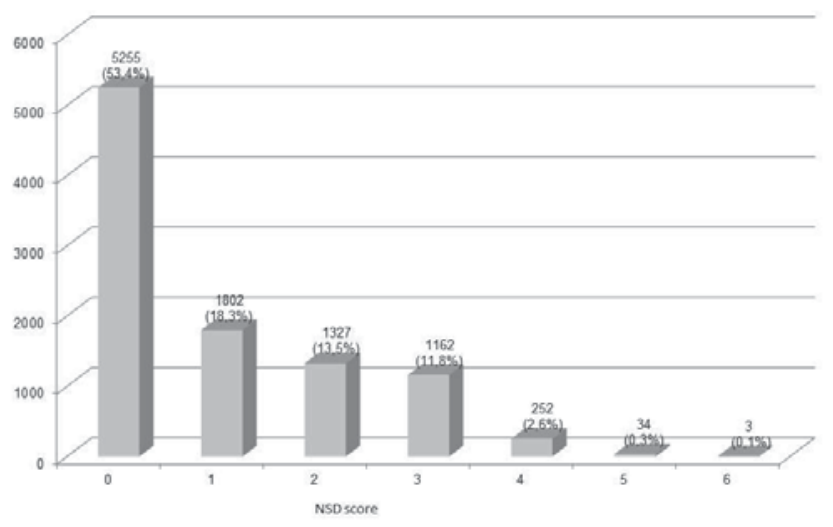

Figure 4. NSD scores obtained after evaluating both nasal cavities together. NSD: Nasal Septum Deviation.

with NSD and 4,762 (90.61\%) of those without NSD were born through spontaneous vaginal delivery. 398 (8.68\%) patients with NSD and 493 (9.38\%) of those without NSD were born after caesarean section. Thus there is no significant relationship between mode of delivery and prevalence of NSD $(p=0.23)$.
No nasal obstruction complaint was given by 6,345 (64.51\%) persons and those scored 0 on the VAS. 3,490 (35.49\%) persons scored their complaints other than zero on the VAS (Figure 2). A total of 1,362 (29.73\%) persons with NSD scored 0 on the VAS and their mean NSD score was 1.2. The mean VAS scores of patients with NSD and ICH, and those with NSD without ICH were 3.51 and 2.39 , respectively. There was a statistically significant difference between mean VAS scores of participants with NSD only and those with NSD and ICH $(p<0.001)$. The mean VAS scores of patients with NSD (without regarding $\mathrm{ICH}$ as present or not), and those with or without NSD were 2.58 and 0.15 , respectively. There was a statistically significant difference between mean VAS scores of participants with NSD (without regarding $\mathrm{ICH}$ is present or not), and those without NSD $(p<0.001)$. These data shows that, although nearly $30 \%$ (29.73\%) of the patients with NSD scored 0 on VAS, there is a strong association between VAS scores and NSD (without regarding $\mathrm{ICH}$ as present or not) (Figure 3).

A total of 1,068 (23.31\%) of participants with NSD scored 5 or higher on VAS and 3,512 (76.69\%) of those with NSD scored 4 or lower values on VAS. Additionally all the patients were scored according to our NSD classification system objectively. Patients were scored varying from 1 to 6 according to severity of their septal deviation (Figure 4). NSD scores of 5,255 (53.43\%) participants were 0. 4,291 (93.69\%) participants with NSD scored between 1 to 3 according to NSD scoring method and their mean VAS score was 2.41. 289 (6.31\%) participants with NSD scored between 4 to 6 and their mean VAS score was 5.20. There was a statistically significant difference between VAS scores of these two groups with NSD $(p<0.001)$.

\section{Discussion}

The normal nasal septum is straight and symmetrical. Factors such as genetic, racial, environmental and trauma have often been identified as the cause of NSD. Generally, NSD is thought to develop at birth, and worsen with age ${ }^{(6)}$. Nasal traumas may happen because of direct nasal trauma as well as abnormal intrauterine posture and difficulties during delivery especially in those without significant nasal trauma history ${ }^{(6-8)}$. Grymer and Melsen suggested that anterior lesions were due to an external cause such as trauma whereas the posterior lesions were due to genetic factors such as the growth of a nasomaxillary complex ${ }^{(9)}$. In 2003, Mladina identified that a type of nasal deformity (type 6 in Mladina's classification) is inherited ${ }^{(10)}$. In another study, $66.66 \%$ of children with nasal injuries had nasal septum deformities, while in a group without injuries, $31.69 \%$ were found to have NSD. In this study, there was statistically significant correlation between NSD and nasal injuries similar to other recent studies ${ }^{(6)}$. 
It has been reported that NSD frequency does not change after the second decade because of an expected decrease in nasal trauma and completed development of the nasomaxillary complex at the end of the second decade ${ }^{(7)}$. Early diagnosis and on time treatment is important in preventing severe nasal septum deformities after nasal trauma ${ }^{(11,12)}$. However, it has also been suggested that there can be delayed results of nasal trauma, and nasal septal deformity can develop until the age of $49^{(13)}$. Patients in our study consisted of young males (mean age was 21.71) in whom the development of the nasomaxillary complex was almost completed. Nasal trauma history was present in $8.40 \%$ of those with NSD, while $0.78 \%$ in those without NSD. According to our results, there was a statistically significant correlation between nasal trauma and prevalence rate of NSD.

NSD precipitates subsequent infections in the paranasal sinuses, upper respiratory tract, and middle ear. It may lead to developmental disorders of the palate and paranasal sinuses. Nasal obstruction is one of the most common symptoms in patients with NSD ${ }^{(7)}$. A systemic NSD classification is required for both surgical planning and clinical trials. There are only a few classification systems developed for this purpose in the literature ${ }^{(1,3-5)}$. However, there is no single classification that has been developed and adopted for everyday clinical use ${ }^{(4,5,14)}$. Mladina defined seven types of septal deviation (four types with vertical deformity, two types with horizontal deformities and another type with atypical deformities) ${ }^{(4)}$. Guyuron et al. defined six types of septal deviation (septal tilt, C-shape anteroposterior deviation, C-shape cephalocaudal, S-shape anteroposterior, S-shape cephalocaudal and localized deviations) ${ }^{(5)}$. Coronal computed tomography has also been utilized to classify NSD ${ }^{(3)}$. These and other classifications schemes were made just according to the shape of the nasal septum. Bauman et al. defined septal deviations using conventional surgical and clinical terms (subluxation of the nasal septum, inclined septum, nasal crest, vomeral spur, height deviation, to the right, to the left, no side deflection, and no specification). This comprehensive classification considers anatomical variations of the inferior and middle turbinates ${ }^{(6)}$.

While evaluating NSD using current classification methods, we identified various pathologies that were not mentioned in the current classification schemes. This may lead to mild pathologies that are either ignored or the pathology is classified as a more severe type that includes combined pathologies. Additionally, current classification systems are not practical for prevalence studies in large patient groups. They are not practical to be able to show the exact relationship between NSD and nasal obstruction either. The classification system we have developed is very practical for evaluating NSD as well as the accompanying $\mathrm{ICH}$, and is very helpful for prevalance studies. It is also useful for determining the relationship between NSD and severity of nasal obstruction.

It has been reported in the literature that anterior deviations are more frequent than posterior deviations and anterior deviations affect the sense of air passage mainly. Assessment of NSD is usually obvious on anterior rhinoscopy to the trained practicioner, however, nasal endoscopy may be required in some cases of posterior deviations ${ }^{(2,10)}$. To focus mainly on anterior deviations, we used direct anterior rhinoscopy for nasal examination in our study. Nasal endoscopy was not performed. Anterior septal deviations were easily evaluated by direct anterior rhinoscopy, however, vasoconstrictive agents and a Killian nasal speculum were used to evaluate posterior septal deviations in some of the patients. 9,284 people were examined in a study that was performed in 60 different areas throughout South Korea by using Mladina's classification. Prevalence of NSD was found to be $22.3 \%$. The frequency of NSD also increased with increasing age; it tended to be predominantly in males and a positive correlation was also found between nasal trauma history and NSD, which was statistically confirmed ${ }^{(13)}$.

In a study performed by Beate and Olga, 288 children with ages ranging 3 to 17 were evaluated for NSD using Mladina's classification. NSD prevalence was found $34 \%{ }^{(6)}$. Subaric and Mladina's study, using the same classification system, demonstrated that the prevalence of nasal septum deformities according to age groups was $28.0 \%$ in the $2-6$ group, $21.1 \%$ in the $7-14$ group, $40.6 \%$ in the $15-18$ group and $41.8 \%$ in the $19-22$ group. In this study, it was also found that NSD was more prevalent in girls than boys ${ }^{(15)}$. On the contrary, another study found that NSD was more frequent in boys. NSD prevalence was $29 \%$ in girls and nearly $37 \%$ in boys. Deformities of the nasal septum in boys could be a result of their physical activity often resulting in injuries ${ }^{(6)}$. Yildirim and Okur in a study of a 1,234 Caucasian children showed that the overall prevalence of NSD was $34.9 \%$. When looking at various age groups, the prevalence of NSD was 16.5 $\%$ in pre-school children, $38.7 \%$ in primary school children and $39.9 \%$ in secondary school children ${ }^{(2)}$. Buyukertan et al., found the rate of NSD in any one region of the nasal cavity among 143 people on coronal computed tomography to be $76.2 \%{ }^{(3)}$. In our study, we found the NSD prevalence to be $46.56 \%$ in young male patients.

Kawalski and Spiewak found an NSD frequency of $22.2 \%$ in children born by spontaneous vaginal delivery, but only 3.9\% among infants delivered by caesarean section. This was a statistically significant difference ${ }^{(7)}$. However, there are studies in the literature that claim no correlation between mode of delivery and NSD ${ }^{(11,16)}$. In our study, we did not found any correlation 
between mode of delivery and NSD existence.

In a prior study, 1,067 patients who underwent septoplasty operation were evaluated and $69.3 \%$ of these patients had unilateral, while $28.5 \%$ of the patients had bilateral ICH. There were only $2.2 \%$ patients that had NSD without accompanying $\mathrm{ICH}^{(1)}$. The frequency of $\mathrm{ICH}$ was $9.1 \%$ among our young male patients, and $17.11 \%$ in those with NSD and $2.11 \%$ in those without NSD. There was a statistically significant correlation between ICH and NSD in our study.

Although NSD is seen quite often, usually it is not severe enough to alter nasal obstruction. VAS is a validated measure that has been widely used in many studies effectively to evaluate patients complaining of nasal obstruction ${ }^{(17-19)}$. The severity of deviation is as important as the location of deviation in evaluating the nasal septum. Septal deviation was evaluated based on stenosis caused by the deviation regardless of being anterior or posterior. In our study, $29.73 \%$ participant scored 0 (no symptom) on VAS, although, they had NSD. $93.69 \%$ of the participants with NSD had a score of 1 to 3 according to our described NSD scoring method and had a mean VAS score of 2.41 . $6.31 \%$ of the participants with NSD had a score of 4 to 6 and had a mean VAS score of 5.20. According to these results, one in three of those with NSD had no nasal obstruction complaint. There were mild septal pathologies in most participants with NSD and even fewer of them had severe septal pathologies that altered nasal function. Presence of both ICH and NSD caused a statistically significant increase in VAS scores. There was a statistically significant correlation between VAS scores and total NSD scores.

In conclusion, we examined a large population of Turkish young males and found that NSD is very frequent and most commonly related to trauma. Subjective patient assessment of NSD varies greatly among patients with similar degree of deviation. Therefore operations have to be designed taking into account the burden of disease on the patient rather than findings examination alone to improve patient perceived outcomes.

\section{Authorship contribution}

MS: Idea for research, planning the methods, data collection, analysis of the results, writing manuscript.

EC: Analysis of the results, writing manuscript, critical review.

AA: Literature search, writing manuscript.

EC: Literature search, writing manuscript.

\section{Acknowlegdement}

We would you like to thank Mrs Ozlem Koksal for statistical analysis and Mrs Gulay Solakoglu for drawings.

\section{Conflicts of Interest}

There is no conflict of interest regarding this study.

\section{References}

1. Baumann I, Baumann H. A new classification of septal deviations. Rhinology 2007 45:220-223.

2. Yildirim I, Okur E. The prevelence of nasal septal daviation in children from Kahramanmaras, Turkey. Int J Pediatr Otorhinolaryngology 2003; 67:1203-1206.

3. Buyukertan M, Keklikoglu N, Kokten G. A morphometric consideration of nasal septal deviations by people with paranasal complaints. A computed tomography study. Rhinology 2003; 41:21-24

4. Mladina R. The role of maxillary morphology in the development of pathological septal deformities. Rhinology 1987; 25: 199-205.

5. Guyuron B, Uzzo CD, Scull H. A practical classification of septonasal deviation and an effective guide to septal surgery. Plast Reconstr Surg 1999; 104:2202-2209.

6. Beate ZJ, Olga SO. The nasal septum deformities in children and adoles cents from Warsaw, Poland. İnt J Pediatr Otorhinolaryngol 2006; 70:731-736.

7. Kawalski H, Spiewak P. How septum deformations in newborns occur. Int J Pediatr Otorhinolaryngol 1998; 44:23-30.

8. Brain D. The etiology of neonatal septa deviations. Facial Plast Surg 1992; 8:191-193.

9. Grymer LF, Melsen B. The morphol- ogy of nasal septum in identical twins. Laryngoscope 1989; 99:642-646.

10. Mladina R, Subaric M. Are some septa deformitites inherited? Type 6 revisited. Int J Pediatr Otorhinolaryngol 2003; 67:12911294

11. Korantzis A, Cardamakis E, Chelidonis E, et al. Nasal septum deformity in the newborn infant during labour. Eur J Obstet Gynecol Reprod Biol 1992; 44:41-46.

12. Sorri M, Laitakari K, Vainio-Mattila J, et al Immediate correction of congenital nasal deformities; follow-up of 8 years. Int J Pediatr Otorhinolaryngol 1990; 19:277-283.

13. Min YG, Jung HW, Kim CS. Prevalence study of nasal septal deformities in Korea: results of a nation-wide survey. Rhinology 1995; 33:61-65.

14. Mladina R, Krajina Z. The influence of caudal process on the formation of septal deformities. Rhinology 1989; 27:113-118.

15. Subariç M, Mladina R. Nasal deptum deformities in children and adolescents: a cross sectional study of children from Zagrep, Croatia. Int J Pediatr Otorhinolaryngol 2002; 63:41-48.

16. Hartikainen-Sorri AL, Sorri M, VainioMattila J et al. Aetiology and detection of congenital nasal deformities. Int J Pediatr Otorhinolaryngol 1983; 6: 83-88.
17. Tahamiler R, Alimoglu Y, Canakcioglu S. Comparison of Odiosoft-Rhino and rhinomanometry in evaluation of nasal patency. Rhinology 2011; 49:41-45.

18. Porter MW, Hales NW, Nease CJ, et al. Longterm results of inferior turbinate hypertrophy with radiofrequency treatment: a new standard of care? Laryngoscope 2006; 116:554-557.

19. Nease CJ, Krempl GA. Radiofrequency treatment of turbinate hypertrophy: a randomized, blinded, placebo-controlled clinical trial. Otolaryngol Head Neck Surg 2004; 130:291-299.

Murat Salihoglu

Otolaryngology Department of GATA Haydarpasa Training Hospital Istanbul Turkey

Tel: +90-534-945 7644

Fax: +90-216-542 2020

E-mail:drmuratsali@gmail.com 